\title{
Des règles aux standards, les enjeux de la régulation de l'école Quelques commentaires critiques à propos de l'article de Walter Herzog
}

\section{Christian Maroy et Vincent Dupriez}

La réforme éducative initiée aujourd'hui en Suisse est appréhendée dans ce texte comme une forme locale de transformations des modes de régulation des systèmes éducatifs qui affectent aujourd'hui l'enseignement dans la grande majorité des pays industrialisés. Dans un contexte d'économie de la connaissance et d'exacerbation de la compétition sur les marchés de l'emploi, la plupart de ces pays sont effectivement en train d'abandonner le modèle de régulation bureaucratico-professionnel au profit de modèles post-bureaucratiques. Dans ceux-ci, quelles qu'en soient les modalités, les standards y révèlent une visée de pilotage par les résultats et une forme renouvelée de normalisation de l'école. Mais la critique de ces formes émergentes ne peut être orientée par l'illusion d'un retour au passé... elle doit surtout permettre d'en rendre les orientations plus citoyennes et moins technicistes, notamment en préservant l'autonomie relative du champ de l'éducation par rapport au pouvoir politique.

La réforme HarmoS en cours dans la confédération helvétique est l'occasion pour Walter Herzog d'interroger une tendance plus large des systèmes éducatifs occidentaux à promouvoir des formes nouvelles d'accountability fondées essentiellement sur la promotion de standards «de compétences» à atteindre par les élèves, les enseignants et les écoles, mais aussi la promotion de diverses formes d'évaluation et de cadrage des pratiques éducatives locales. Dans ce texte, nous reformulerons brièvement la substance de la critique de Herzog, avant de proposer trois réflexions qui la prolongent, la développent, la reformulent sans en remettre en cause fondamentalement le bien-fondé.

Quel est en substance le propos d'Herzog? La réforme des «standards» véhicule une conception réductionniste (de nature économique et technologique) de l'éducation dans ses visées, ses objectifs et ses processus. Elle témoigne d'une conception industrielle, standardisée, rationnalisée des tâches éducatives, qui remettent en cause la conception plus artisanale jusque-là prédominante. Cette conception réductionniste, et plus largement la réforme des systèmes éducatifs qu'elle charrie, va de pair avec deux autres formes de réduction. 
- Une réduction instrumentaliste des sciences de l'éducation désormais vouée à «instrumenter» la politique éducative, en développant les fondements théoriques des standards, les modèles de compétences auxquels ils renvoient, en assurant aussi l'analyse des fonctionnements et processus au sein du système éducatif, de façon à orienter des formes de rétroaction et de correction du système.

- Une réduction potentielle de la professionnalité enseignante qui risque de voir son rôle et son intervention de plus en plus balisée par les résultats d'une science de l'éducation, rabougrie et amputée de sa dimension critique.

Ces évolutions vont de pair avec une forme de mise sous tutelle et de perte de pouvoir des scientifiques par rapport au politique, alors qu'un processus analogue risque aussi de limiter l'autonomie professionnelle enseignante.

Cette critique de Herzog est une critique interne au champ des sciences de l'éducation plutôt qu'une critique qui s'élaborerait d'un point de vue extérieur. Elle s'appuie en effet sur une conception «humaniste» et "artisanale» de l'éducation, de ses visées et modes de fonctionnement pour s'opposer à des réformes dont il brocarde les dérives «technocratiques» 1 .

Cette critique est fondée, s'agissant du rôle des sciences de l'éducation, sur la mise en avant de l'illusion scientiste et positiviste d'une vérité scientifique qui se soutiendrait des seules évidences empiriques, par opposition à une conception du travail scientifique reposant davantage sur des arguments venant étayer des propositions théoriques. S'agissant de la conception de l'éducation, elle se fonde sur l'idée (largement diffusée) que les processus éducatifs sont "sous-déterminés technologiquement». Ainsi pour de nombreux théoriciens des organisations comme pour Herzog (voir notamment Meyer \& Rowan, 2006), les «technologies» de l'enseignement sont très incertaines et on ne maîtrise pas aujourd'hui les relations entre les moyens et techniques utilisés et les résultats obtenus. Les processus éducatifs sont ainsi des processus de communication entre sujets, qu'on ne peut ramener sans réduction à un processus mécanique de transformation d'un objet, à une " production d'outputs ", adoptant ainsi la métaphore de la production industrielle, appréhendée sous le seul angle de la mesure quantitative.

Ainsi, pour reprendre la grammaire proposée par Boltanski et Thevenot (1991), pour analyser les formes de «dénonciation» des «injustices» ou "désordres» sociaux, la critique de Herzog est une critique d'une vision du monde de l'éducation appréhendé dans les catégories de la "cité industrielle», alors que sa propre vision s'ancre davantage dans un monde qui participe sans doute tantôt de la cité «civique» (par exemple en accentuant la nécessité d'une science critique et pas seulement instrumentale) tantôt d'une cité "domestique» ou communautaire (insistant sur les relations et valeurs partagées dans des relations inter-subjectives).

Nous voudrions à présent proposer trois réflexions, qui ne remettent pas fondamentalement en cause les critiques de Herzog, mais s'efforcent d'en sociologiser la portée. Nous nous efforcerons dès lors de prendre un point de vue critique 
davantage externe dans le but d'éclairer quelque peu les significations sociales et politiques des transformations des modes de gouvernance scolaire.

\section{Première réflexion}

À notre sens, les transformations du pilotage auxquelles on assiste en Suisse, comme dans nombre d'autres pays européens (voir Maroy, 2006) sont symptomatiques de changements dans le régime dominant de régulation des systèmes éducatifs ${ }^{2}$. Le modèle «bureaucratico-professionnel» (qui sera parfois appelé modèle bureaucratique dans la suite de ce texte) de régulation des systèmes éducatifs avait accompagné, avec d'importantes variantes nationales, la construction et le développement de systèmes éducatifs nationaux de masse dès les années 50/60. Dans ce modèle, la régulation et le contrôle étaient donc basés sur des arrangements tels que le contrôle de conformité aux règles, la socialisation et l'autonomie de professionnels de l'éducation ou la régulation conjointe (État/syndicats enseignants) de questions d'emploi, mais aussi du curriculum. Ce modèle de régulation est désormais travaillé par des politiques éducatives qui tendent à substituer ou superposer à ces anciens modes de régulation de nouveaux arrangements institutionnels post-bureaucratiques basés soit sur le modèle du quasi-marché (Bartlett \& Legrand, 1993) et/ou sur le modèle de l'État évaluateur (Broadfoot, 2000) (pour plus de détails sur ces modèles, voir Maroy 2006).

Le passage d'un pilotage par l'input à un pilotage par l'output, est fondé sur une responsabilisation des professionnels soit par rapport à des usagers, soit par rapport à l'État. Alors que le quasi-marché responsabilise par la pression du marché et de la compétition (market based accountability), l'État évaluateur responsabilise vis-à-vis de l'État ou de ses représentants (government based accountability). En effet, l'État évaluateur fixe désormais de façon beaucoup plus précise des objectifs standards et minimaux que le système éducatif doit atteindre. Simultanément, il accorde une autonomie accrue aux écoles (décentralisation) en matière pédagogique ou gestionnaire, tout en mettant en place de nouveaux outils "d'évaluation» des résultats de ces entités décentralisées, à travers des dispositifs de mesure externe, des acquis des élèves en particulier. De nouveaux instruments d'action publique basés sur les connaissances se mettent en place: évaluations statistiques, mais aussi "benchmarking» et repérage des «bonnes pratiques», mise en place de dispositifs de diffusion et de transmission de pratiques «efficaces» par la formation et la supervision. Par ailleurs, la régulation n'est plus seulement fondée sur des normes sociales et professionnelles, relayées à travers la socialisation et la formation professionnelle, et fondatrices de l'identité et d'une forme d'homogénéité normative et cognitive du corps enseignant. Ces normes deviennent progressivement des "normes techniques», qui se revendiquent d'un savoir neutre et objectif, et qui sont diffusées ensuite par des conseillers et experts en pédagogie. 
En bref, l'État évaluateur (ou par ailleurs le quasi-marché), participe d'un nouveau régime de régulation post-bureaucratique qui s'oppose au modèle bureaucratique, pour plusieurs raisons. Tout d'abord, le modèle bureaucratique est une forme d'organisation et de coordination qui s'appuie sur une valorisation de la loi, de la raison, lesquelles en fondent la dimension institutionnelle. Cela signifie que le caractère bureaucratique n'est pas seulement de nature instrumentale et organisationnelle; la dimension bureaucratique de l'école participe de la forme scolaire, qui institue la médiation de la règle comme dimension socialisatrice fondamentale (Vincent, Lahire \& Thin, 1994). Et comme l'a relevé Derouet (1992), le recours intensif à la règle comme forme d'organisation du champ scolaire doit aussi être lue comme l'expression de la volonté politique de déployer et d'opérationnaliser le principe d'égalité de traitement des élèves. Autrement dit, l'apprentissage de la règle et selon les règles, dans un cadre impersonnel et bureaucratique, revêt une dimension simultanément organisationnelle et institutionnelle. La forme bureaucratique participe de ce que F. Dubet appelle ainsi le programme institutionnel de l'école moderne (Dubet, 2002), en phase avec d'autres formes comme l'État.

À l'opposé, les modèles post-bureaucratiques se présentent comme value free. Ils se donnent à voir comme de pures formes organisationnelles rationnelles, qui peuvent prétendre se mettre au service de finalités variables, comme la promotion autant de l'équité que d'une scolarisation de qualité, finalités exogènes au mode de régulation qui se veut pure instrumentalité. L'important est de mettre en place "ce qui marche», ce qui "produit des résultats». Ainsi, ces deux modes de régulation constituent une première rupture par rapport à la forme bureaucratique en ce sens qu'ils valorisent la seule rationalité instrumentale, l'efficacité et la performance sans en apparence engager de rationalité axiologique intrinsèque. Ils imposent une pression aux systèmes, aux organisations et aux professionnels pour que ceux-ci contribuent à produire des résultats tangibles, visibles et mesurables. En ce sens, l'univers normatif de référence est celui d'une performativité de plus en plus auto-référencée et de moins en moins renvoyée à un fondement institutionnel externe.

Une autre différence tient aux instruments d'action publique mobilisés pour produire un effet de régulation. Ainsi les modes de contrôle présents sont beaucoup plus étendus (ou intrusifs) que le contrôle de conformité aux règles, dominant dans le modèle bureaucratique. Ils impliquent désormais de nouveaux «outils» d'évaluation (évaluation standardisée des acquis scolaires, tableaux de bord, indicateurs, mais aussi les rapports, les bilans etc.), ainsi que la définition de procédures permettant de définir diverses «bonnes pratiques» en référence aux résultats visés. Ces outils et procédures, apparemment seulement techniques, sont producteurs de normes, relayées à travers des programmes de formation et de supervision, pratiques qui peuvent aboutir à un processus d'enrôlement des acteurs de base. Des outils sont ainsi développés en complément des règles, qui persistent et démontrent la filiation des modèles post-bureaucratiques avec le 
modèle bureaucratico-professionnel. La palette d'instruments (au sens de Lascoumes \& Le Galès, 2004) de la régulation post-bureaucratique s'est ainsi élargie par rapport à la régulation bureaucratique: la régulation bureaucratique s'appuyait essentiellement sur des instruments législatifs et réglementaires, là où la régulation post-bureaucratique s'appuie aussi sur des instruments «conventionnels et incitatifs» (comme la gouvernance par contrat), et la construction de normes et standards de référence, par rapport auxquels le travail d'évaluation va prendre son sens.

Un troisième élément qui distingue les modes de régulation post-bureaucratiques du mode de régulation bureaucratico-professionnel, est lié à la distribution du pouvoir entre acteurs. Si la régulation bureaucratico-professionnelle ménageait un équilibre entre le pouvoir de l'État et celui du groupe professionnel enseignant, au détriment sans doute du poids pris par les usagers et les parents, les nouveaux modes de régulation post-bureaucratiques tendent à limiter le pouvoir des professionnels, soit au bénéfice des usagers (quasi-marché) soit au bénéfice de l'État.

En bref donc, Herzog a raison de s'inquiéter des changements en cours, car il ne s'agit pas seulement de changements techniques, concernant les modes de contrôle et de coordination d'une organisation (passage du pilotage input au pilotage output). Ce changement s'inscrit dans d'autres changements de fond, de nature institutionnelle et normative, dont il a bien perçu la portée, et qui, nous semble-t-il, remettent en cause les fondements institutionnels et organisationnels de la bureaucratie, que Max Weber, associait à un mode de légitimation du pouvoir, fondé sur la loi et la raison. Un aspect que la métaphore de l'artisanat et de l'industrie ne capte pas $^{3}$.

\section{Deuxième réflexion}

Une question non posée par Herzog est alors de savoir pourquoi dans divers pays européens (et ailleurs), les modèles de régulation sont en train d'évoluer dans ce sens. L'argument invoqué par les politiques est souvent que les systèmes éducatifs ne sont pas assez efficaces et/ou pas assez équitables (notamment par référence à d'autres systèmes, que des études internationales comme Pisa permettent de mettre en balance). Un tel discours peut chercher à enrôler et à convaincre tant les professionnels que l'opinion publique de la légitimité des changements à effectuer. On ne peut cependant limiter les facteurs de changement à ces argumentaires discursifs. Il nous semble que les processus sociaux engagés dans ces changements sont de plusieurs natures.

- Les transformations technico-économiques (montée du post-fordisme ou d'une "économie de la connaissance»), les nouvelles formes de compétition économiques (reposant autant sur la qualité, les innovations techniques, les services associés aux produits, et pas seulement sur les prix) tout comme la 
globalisation de la concurrence économique et financière, conduisent les États à pondérer différemment leurs politiques. Les politiques macro-économiques nationales sont nettement plus limitées qu'avant (politique monétaire, politique de la demande...) si bien que nombre d'entre eux ont renforcé les politiques dites de «'offre», qui peuvent tantôt porter sur les facteurs de compétitivité "prix» à court terme, tantôt consister à agir sur le "capital humain» et le marché du travail propre à chaque région ou nation. Dans la compétition mondiale, et dans le nouveau paradigme productif émergent, il est de plus en plus vital de développer non seulement des politiques d'éducation et de formation, mais aussi de recherche-développement. Ces politiques conduisent les États à faire des systèmes éducatifs des «auxiliaires» dans leurs politiques de positionnement économique. La stratégie européenne dite de Lisbonne trouve là son fondement. Dans ce contexte en effet, les États cherchent à «améliorer» les performances des systèmes éducatifs, de plus en plus conçus comme finalisés par la production d'un «capital humain», là où ils étaient perçus naguère comme des institutions fondatrices de l'État nation. Au moment où les États nations sont fragilisés par la mondialisation, tout se passe comme si l'institution scolaire changeait progressivement de fonction et de signification.

- L'école a toujours eu un rôle dans la reproduction des inégalités sociales et la distribution des nouvelles générations dans les positions différenciées et hiérarchisées de la division sociale du travail. Elle est également le lieu de compétition entre les différents groupes sociaux pour l'appropriation d'un capital culturel distinctif, qui leur permette d'assurer un positionnement social et professionnel. Or dans le contexte de précarité et de chômage structurel important qui est le nôtre, dans le régime de compétition accrue qui se développe sur le marché du travail, les classes moyennes sont de plus en plus anxieuses par rapport à l'avenir de leurs enfants et cela alimente une "demande sociale" pour une éducation de "qualité», qui permette de garantir l'avenir. Une demande de différenciation des parcours et des institutions scolaires également, qui permette au «bien éducatif» de rester un bien dont la valeur est positionnelle. Ainsi, peut-on rendre compte de la demande sociale pour «un plus grand choix» des établissements par les parents, possibilité qui leur permette d'éviter les contextes éducatifs dont le niveau scolaire ou les conditions de discipline et de socialisation leur apparaissent trop problématiques; de même, la demande sociale de "qualité» adressée aux écoles et aux enseignants trouve dans l'inquiétude des classes moyennes pour leur propre destinée sociale un de ses terreaux structurels. Ces inquiétudes peuvent être aussi avoir des répercussions dans les programmes politiques des partis et les enjeux électoraux, et se retrouver in fine dans une volonté plus affirmée de l'État de contrôler le système éducatif et les enseignants.

- Un dernier facteur important de convergences des politiques éducatives tient à divers mécanismes de transfert et de dissémination des modèles qui inspi- 
rent à des degrés divers les politiques éducatives. On a vu dans ces mécanismes les effets d'un processus d'apprentissage et d'emprunt des politiques (Halpin \& Troyna, 1995) voire de contamination (Levin, 1998). Plus largement, on peut avancer que divers acteurs collectifs ou individuels (organisations internationales, académiques, experts en politiques éducatives) contribuent à transmettre concrètement des modèles de référence en matière de régulation et de gouvernance des systèmes (Ball, 1998; Normand, 2003).

Cette analyse aide à voir pourquoi l'État cherche à agir de façon plus intrusive qu'auparavant sur les institutions éducatives. Les nouveaux modes de régulation post-bureaucratiques, évoqués jusqu'ici, et dénoncés par Herzog, cherchent à réussir là où les politiques éducatives antérieures ont régulièrement échoué. C'est en effet un vieux constat de la recherche en éducation que d'avoir souligné à quel point les réformes des systèmes d'enseignement peinent à transformer les pratiques pédagogiques et s'accompagnent le plus souvent d'un conformisme de façade. Dans la même veine, les analyses des établissements scolaires ont souligné à quel point les établissements sont des systèmes faiblement couplés et caractérisés par une très large autonomie de chaque enseignant au sein de sa classe. En quelque sorte, ces processus de régulation post-bureaucratiques renouent, par des stratégies renouvelées, avec cette ambition de "peser» sur les pratiques enseignantes, pour les transformer. Les changer pour améliorer l'efficacité et la qualité des systèmes, parfois leur équité. Mais sur un fond de performativité que nous avons déjà évoqué.

Face à ces changements, les acteurs scolaires et les chercheurs en sciences de l'éducation n'ont peut-être pas une multitude de réponses ou de réactions possibles. Soit épouser le mouvement avec enthousiasme, en espérant peut-être y trouver un bénéfice, soit s'y opposer de façon défensive et nostalgique, en espérant maintenir au maximum l'institution scolaire (mi-réelle, mi-rêvée) du passé, soit encore chercher à modaliser ou à infléchir la trajectoire des changements en cours. Et ceci nous conduit à notre troisième réflexion.

\section{Troisième réflexion}

Notre analyse suggère donc que les rapports de pouvoir et l'évolution de l'environnement scolaire sont tels qu'on ne peut éviter complètement un changement des modes de régulation des systèmes d'enseignement. La possibilité d'améliorer la qualité et l'équité des systèmes éducatifs, à travers ces nouveaux modes de gouvernance, ne peut non plus être négligée, même si la démonstration incontestable de tels effets reste encore à produire. La question fondamentale est dès lors d'identifier quels sont les infléchissements et les contre-pouvoirs nécessaires afin d'éviter le double réductionnisme identifié par Herzog qui menace simultanément les sciences de l'éducation et la professionnalité des enseignants. Nous ébauchons ci-dessous quelques éléments de réponse à cette double interrogation. 
Avec Herzog, nous ne pouvons bien entendu que plaider pour l'autonomie scientifique d'un champ de recherche qui doit préserver sa fonction critique. Mais, cela ne doit pas empêcher les chercheurs en éducation de suivre de près les transformations politiques discutées ici, et d'en identifier notamment les effets sur les élèves, les enseignants et les systèmes scolaires. De telles recherches doivent mener, chaque fois que cela est possible, à la construction de relations entre les paramètres étudiés et si de telles recherches font apparaître que certaines modalités pédagogiques ou organisationnelles s'accompagnent des effets souhaités, il s'agira bien sûr d'une avancée en termes de connaissances. Mais de telles recherches ne peuvent servir à alimenter mécaniquement des politiques qui seraient fondées sur des évidences (evidence-based policy). En d'autres mots, la science ne peut en aucun cas être réduite à la production de données empiriques conformément à des catégories définies par d'autres et le politique ${ }^{4}$ ne peut non plus être réduit à l'usage mécanique de telles données. Car les résultats de comparaisons empiriques ne peuvent se traduire automatiquement en politiques et en règles de conduite. Et si la science est susceptible d'informer une pratique socialement constituée, par contre elle ne la "fonde» pas et ne peut servir à la justifier. Ces fonctions relèvent de valeurs et de normes, irréductibles à des énoncés scientifiques (voir Lessard, 2007). Par ailleurs, une science digne de ce nom vise surtout à produire de l'intelligibilité, c'est-à-dire des modèles compréhensifs ou explicatifs qui permettent de rendre compte des phénomènes appréhendés et observés. Et un tel travail scientifique suppose notamment que les chercheurs soient capables de définir leurs objets propres, d'interroger et de discuter leurs modèles et leurs outils, ce qui ne peut se faire qu'en garantissant une autonomie suffisante du champ scientifique vis-à-vis du champ politique.

Plus fondamentalement, comme Weber l'a montré il y a longtemps déjà dans le Savant et le Politique (1919/1963), ce n'est pas parce que tout objet en sciences sociales implique inéluctablement un rapport aux valeurs, qu'il faut en prendre prétexte pour balayer toute idée de vérité comme idéal régulateur du monde scientifique et subordonner toute recherche à sa valeur dans le registre de l'action, à sa valeur performative. La connaissance est aussi un bien et elle ne peut, dans une société pluraliste et démocratique, être subordonnée aux seules fins définies par leurs dirigeants, fussent-ils démocratiquement désignés. Les sciences de l'éducation, comme d'autres sciences sociales, ne peuvent être réduites de façon unilatérale à une perspective instrumentale de gestion, de "réforme», ou d'action sur le monde. Elles engagent toujours une réflexion herméneutique et critique sur le sens de l'action et sur les finalités sociales et subjectives de nos existences et mondes sociaux. C'est pour cela aussi, qu'il est si important de maintenir une autonomie du champ scientifique, car elle est le gage d'un recul critique qui est une valeur en soi, par-delà sa contribution à l'efficacité scientifique. L'exercice de la raison scientifique ne peut, dès lors, être réduit à la rationalité instrumentale. 
Les craintes exprimées par Herzog à propos de la professionnalité des enseignants nous semblent également légitimes. En effet, si le déploiement du modèle de l'État évaluateur vient consolider la position des autorités publiques, comme nous l'avons exposé ci-dessus, l'opérationnalisation d'un tel modèle vient simultanément renforcer un corps important d'experts et de techniciens, qui sont chargés de concevoir les instruments de la régulation: les standards, les tests, les indicateurs... et qui contribuent de la sorte à un travail de normalisation du champ scolaire. Dans un tel processus, les enseignants se retrouvent dès lors de facto dans une position d'exécutants, position assez paradoxale à l'heure où la professionnalisation de leur métier est proclamée de toute part. À l'encontre d'une telle évolution, et même si cela s'accompagne nécessairement d'un travail plus long et plus complexe, il nous semble indispensable d'associer les enseignants à la conception de telles réformes et à la (re)définition de leur propre métier. Car ces nouveaux curricula ne représenteront un défi positif pour les enseignants que s'ils sont perçus comme des dispositifs légitimes et significatifs. Un des enjeux majeurs des évolutions commentées ici est donc bien la place qui est faite aux enseignants comme corps professionnel. Il importe donc de réfléchir à la façon dont les différents segments d'un corps enseignant, en vérité assez fragmenté, pourraient constituer un acteur collectif, à la façon dont, dans le passé, il s'est trouvé être un acteur important, allié aux élites politiques pour construire l'institution scolaire.

Ces quelques lignes ne représentent bien sûr qu'une ébauche de réponse, mais elles ont surtout pour ambition de contribuer à alimenter un débat que la critique de Herzog ouvre de manière salutaire. Accepter de poser et d'assumer ces interrogations nous semble primordial pour éviter la déprime et la nostalgie, ou la naïveté béate et optimiste face aux changements en cours.

\section{Notes}

1 Cette critique peut ainsi être interprétée comme une intervention au sein des luttes internes au champ de l'éducation, à propos des finalités et des méthodes éducatives. Ainsi, elle s'adresse non seulement aux politiques mais peut-être surtout aux acteurs du champ de l'éducation (scientifiques ou praticiens) qui seraient tentés par une politique de changement éducatif, fondé sur l'accountability et les standards.

2 Les modes de régulation institutionnels d'un système éducatif peuvent être considérés comme l'ensemble des mécanismes d'orientation, de coordination, de contrôle des actions des établissements, des professionnels ou des familles au sein du système éducatif, modes de régulation mis en place par les autorités éducatives grâce à différents arrangements institutionnels (règles, lois, structures de pouvoir, dispositifs d'information, d'évaluation ou encore de marché). Ces modes de régulation institutionnels ou politiques ne constituent pas le tout de la régulation, dans la mesure où plus largement la régulation sociale procède de régulations autonomes produites au quotidien par les acteurs de «base» mais aussi de paradigmes, «modèles» cognitifs et normatifs qui constituent les cadres dans lesquels les acteurs appréhendent et définissent leur situation, tant dans le «haut» que le «bas» du système.

3 Par ailleurs, et nous n'aurons pas le temps de le développer ici, les nouveaux modes de régulation en gestation pourraient aussi s'avérer post-professionnels, un aspect que Herzog discute sans le développer (voir à ce sujet Maroy, 2008). 
4 Le politique signifie ici la capacité, proprement politique, à décider et mettre en œuvre des «politiques» (policies).

\section{Références bibliographiques}

Ball S. J. (1998). Big Policies/Small World: an introduction to international perspectives in education policy. Comparative Education, 34 (2), 119-130.

Bartlett, J. \& Legrand, W. (1993). The theory of quasi-markets. In J. Legrand \& W. Bartlett, Quasi-Markets and Social Policy (pp. 13-34). Houndmills: MacMillan Press.

Boltanski, L. \& Thevenot, L. (1991). De la justification: Les économies de la grandeur. Paris: Gallimard.

Broadfoot, P. (2000). Un nouveau mode de régulation dans un système décentralisé: l'État évaluateur. Revue française de pédagogie, 130, 43-55.

Derouet, J. L. (1992). École et justice. Paris: Métailié.

Dubet, F. (2002). Le déclin de l'institution. Paris: Seuil.

Halpin D. \& Troyna B. (1995). The politics of education policy borrowing. Comparative Education, 31 (3), 303-310.

Lascoumes, P. \& Le Galès, P. (Éd.). (2004). Gouverner par les instruments. Paris: Presses de la Fondation nationale des sciences politiques.

Levin B. (1998). An epidemic of education policy: (what) can we learn from each other? Comparative Education, 34 (2), 31 - 141.

Lessard, C. (2007). Les usages politiques de la recherche en éducation. In V. Dupriez \& G. Chapelle (Éd.), Enseigner (pp. 69-79). Paris: PUF.

Maroy, C. (2006). École, régulation, marché. Une comparaison de six espaces scolaires locaux en Europe. Paris: PUF.

Maroy, C. (2008). Régulation post-bureaucratique des systèmes d'enseignement et travail enseignant. Conférence au 20e colloque de l'ADMEE-Europe, Genève, Université, 9-11 janvier 2008.

Meyer, H. D. \& Rowan, B. (Éd.). (2006). The new institutionalism in education. New-York: State University of New York Press.

Normand, R. (2003). Les comparaisons internationales de résultats: problèmes épistémologiques et questions de justice. Education et Sociétés, 12 (2), 73-89.

Vincent, G., Lahire B. \& Thin D. (1994). Sur l'histoire et la théorie de la forme scolaire. In G. Vincent (Éd.), L'éducation prisonnière de la forme scolaire? Socialisation et scolarisation dans les sociétés industrielles (pp. 11-50). Lyon: Presses Universitaires.

Weber, M. (1919). Le Savant et le politique (Édition française 1963). Paris: Union générale d'Éditions.

Mots clés: standards, régulation, évaluation, professionnalisation 